\title{
Instruction Hints for Super Efficient Data Caches
}

\author{
Jie Tao $^{1}$, Dominic Hillenbrand ${ }^{2}$, and Holger Marten ${ }^{1}$ \\ ${ }^{1}$ Steinbuch Center for Computing \\ Forschungszentrum Karlsruhe \\ Karlsruhe Institute of Technology, Germany \\ \{jie.tao, holger.marten\}@iwr.fzk.de \\ ${ }^{2}$ Computer Laboratory \\ University of Cambridge, United Kingdom \\ dh378@cam.ac.uk
}

\begin{abstract}
Data cache is a commodity in modern microprocessor systems. It is a fact that the size of data caches keeps growing up, however, the increase in application size goes faster. As a result, it is usually not possible to store the complete working set in the cache memory.

This paper proposes an approach that allows the data access of some load/store instructions to bypass the cache memory. In this case, the cache space can be reserved for storing more frequently reused data. We implemented an analysis algorithm to identify the specific instructions and a simulator to model the novel cache architecture. The approach was verified using applications from MediaBench/MiBench benchmark suite and for all except one application we achieved huge gains in performance.
\end{abstract}

Keywords: Cache optimization, simulation, architecture design.

\section{Introduction}

The memory system is a traditional optimization target for enhancing the overall performance of a computational architecture. With the microprocessor design increasingly focusing on the power issue, memory also becomes the goal of optimization in terms of both performance and energy consumption.

To optimize the memory system, cache is the key. In the performance aspect, an access to the cache is much more efficient than a reference to the main memory because a cache access takes only several CPU cycles while an access to the main memory needs more than 100 cycles on modern processors. Hence, a lower cache miss rate can reduce the CPU time for executing an application. This actually also decreases the power requirement because accessing the main memory consumes more energy than accessing the cache.

The common approach for cache optimization focuses on improving the data locality so that data stored in the cache can be reused before being moved back to the main memory. This locality is typically achieved by restructuring the program code based on affinity analysis [81012 1314]. 
We propose a different approach that enhances the cache efficiency, in terms of both performance and energy consumption, by storing only carefully selected data in the cache. We achieve this goal with an analysis algorithm that goes through the memory access trace of an application and thereby detects load/store instructions which do not introduce any cache hits. We decide not to load the data required by these instructions into the cache. In this case, cache space can be maintained for other data.

We then implemented a cache simulator for verifying the proposed approach and also for providing researchers a generic tool to study the impact of cache bypassing on the performance of applications. The simulator models the basic functions of a traditional cache with an additional functionality of bypassing the data of specific instructions.

The MediaBench and MiBench benchmarks for embedded system were used to examine the results of our cache optimization. As the cache is usually small on embedded systems it is more valuable to use their cache space efficiently. For the tested applications we achieved an average improvement of $2.7 \mathrm{x}$ in cache hit rate.

The remainder of the paper is organized as following. Section 2 first gives an overview of related work on cache optimization. This is followed by a detailed description of the proposed concept in Section 3 . Section 4 presents the initial experimental results. The paper concludes in Section 5 with a short summary and several future directions.

\section{Related Work}

In the area of cache optimization, a lot of research work has been performed and different approaches were proposed. They either only address the performance aspect or also have specific mechanisms for power saving. Here, we focus on the latter.

Esakkimuthu et al. 5] deployed both hardware and software techniques to save the energy consumption of the memory system. The hardware approach includes a block buffering scheme and a sub-banking strategy. The former uses a buffer to store the previously accessed cache line. In this case, if the next data request targets on the same cache line, data can be acquired from the buffer without accessing the cache. In the sub-banking optimization, the data array of the cache is partitioned into banks and by a data request only the corresponding bank is accessed rather than the whole cache. The software approach applies the conventional code optimization schemes like loop interchange, loop tiling, and loop unrolling to improve the data reuse with a combined result of less cache misses and energy consumption.

Yang et al. [16] proposed a cache architecture to reduce the power demand but without performance degradation. This architecture deploys an L0, an instruction cache between CPU and the L1 instruction cache, to store frequently accessed instructions. A steering mechanism is employed to direct an instruction to the correct location. Simulation results show a $52 \%$ instruction cache energy reduction on average for a set of multimedia applications. 
Benitez et al. 3] proposed a reconfigurable cache architecture that can be adapted to the running programs. The adaptation scheme is based on two techniques: a learning process provides the best cache configuration for each program phase, and a recognition process detects program phase changes. In addition, a low-overhead reconfiguration mechanism was designed. Simulation results show that this approach can achieve performance improvement and cache energy saving at the same time. Similarly, Cordon-Ross et al. 6] also address reconfigurable cache architectures but use heuristic tuning method to determine the best cache parameters. A specific contribution of this work is its mechanisms for tuning a data and instruction unified second level cache. As such tuning covers a large space, this approach has achieved a higher power saving than the other one. Abella et al. 1 also focus on the second level cache and designed a hardware technique to turn off those cache lines that are not expected to be reused. Alternatively, Ishihara et al. 9] use specific algorithm to place the code in the cache in a way that not all cache lines in a set are used. Unused cache lines can be disconnected for saving the power.

Overall, various schemes have been deployed in the last years for performance and energy issues of the memory system. They can be roughly classified to two groups: one focuses on improving the data locality with additional hardware support and the other on adapting the cache architecture to applications. Our approach can be classified to the first group because it also addresses on the locality issue. However, this approach is different from other work in that it allows some data accesses to bypass the cache memory so to save spaces for more important data.

\section{The Ihint Architectural Approach}

Key to our performance improvements is a novel cache architecture capable of bypassing the data of some loads/stores according to the instruction hints (Ihint for short). The instructions are selected by an analysis algorithm and the cache architecture is simulated.

\subsection{The Ihint Cache}

Figure 1 shows the proposed cache architecture which is a slightly modified version of the traditional one. We mainly extend the ways of data transferred between the processor and the off-chip memory. In comparison to a standard cache design, we allow direct reads by the processor and provide a FIFO for potentially non-blocking writes. In both case the cache is bypassed and its state is untouched. Accesses to the off-chip memory are controlled by the arbiter.

The cache is accessed in case of a hit. In case of a miss, the actions depend on whether a special-instruction has been encountered. A special-instruction always bypasses the data cache and leads to a direct read from off-chip memory or to a write into the FIFO. 


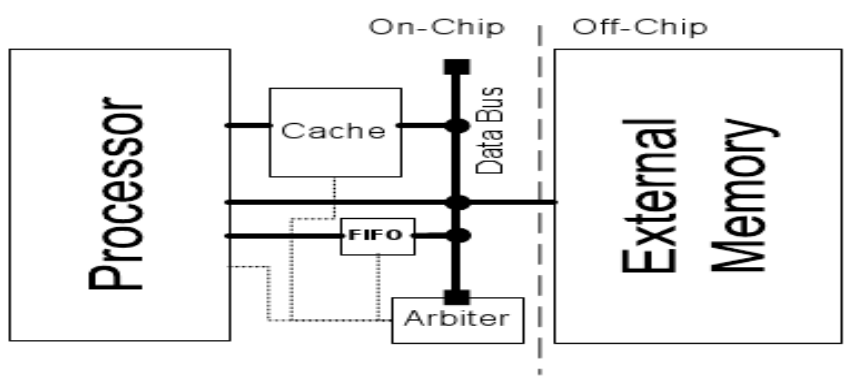

Fig. 1. Ihint-Cache Overview

\section{2 "Special"-Instruction Selection Process}

The hardware provides us with more choices in the memory paths. Now we need to decide which load/store-instructions can be regarded as "special". For this, an analysis algorithm is designed and implemented.
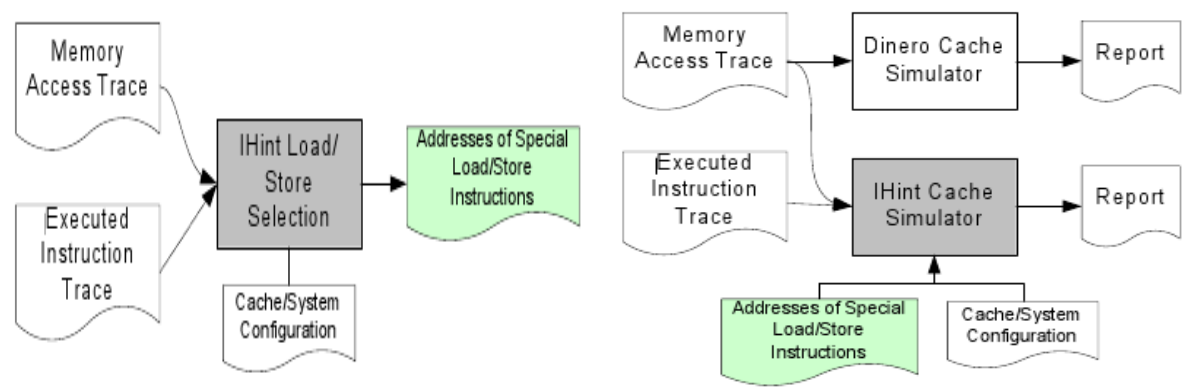

Fig. 2. Instruction selection and cache simulation

The left diagram in Fig. 2 illustrates the workflow of the analysis process. As depicted in the diagram, the analysis algorithm needs a memory access trace and an instruction trace as input. We acquire both trace files with the ArchC [2] Instruction Set Simulator. For recording the executed instruction and data access addresses we modified this simulation tool.

The memory access and instruction traces allow us to determine which memory accesses go along with a distinct instruction. This is essential for the analysis algorithm to look for special load/store instructions.

Selecting special instructions is actually to decide whether to move data from off-chip memory to the cache at a read/write miss. The analysis is split into two distinct steps. One for identifying the special loads and one for the stores. The analysis in both steps is based on the difference between the amount of cache hits and misses caused by an individual instruction. 
Table 1. Binary instruction break-down by application and architecture

\begin{tabular}{|c|c|c|c|c|c|c|c|}
\hline & \multicolumn{7}{|c|}{ Instructions } \\
\hline & & \multicolumn{2}{|c|}{ MIPS } & \multicolumn{2}{|c|}{ SPARC } & \multicolumn{2}{|c|}{ PowerPC } \\
\hline \multirow[t]{3}{*}{ PEGWIT } & total instructions & \multicolumn{2}{|c|}{23149} & \multicolumn{2}{|c|}{20652} & \multicolumn{2}{|c|}{22405} \\
\hline & loads/stores & $\overline{3524}$ & 2367 & 2913 & 1717 & 3664 & 2809 \\
\hline & special loads/stores & 41 & 149 & 25 & 80 & 53 & 174 \\
\hline \multirow[t]{3}{*}{ CJPEG } & total instructions & \multicolumn{2}{|c|}{36043} & \multicolumn{2}{|c|}{32008} & \multicolumn{2}{|c|}{35388} \\
\hline & loads/stores & 7218 & 4832 & 6353 & 3548 & 7.171 & 5.165 \\
\hline & special loads/stores & 80 & 766 & 67 & 417 & 93 & 821 \\
\hline \multirow[t]{3}{*}{ GSM } & total instructions & \multicolumn{2}{|c|}{21899} & \multicolumn{2}{|c|}{19888} & \multicolumn{2}{|c|}{21124} \\
\hline & loads/stores & $\overline{3289}$ & 2397 & 2660 & 1760 & 3508 & 2682 \\
\hline & special loads/stores & 18 & 46 & 12 & 38 & 38 & 71 \\
\hline \multirow[t]{3}{*}{$\overline{\text { DIJKSTRA }}$} & total instructions & \multicolumn{2}{|c|}{13251} & \multicolumn{2}{|c|}{12100} & \multicolumn{2}{|c|}{13753} \\
\hline & loads/stores & 1944 & 1500 & 1462 & 1008 & 2130 & 1813 \\
\hline & special loads/stores & 11 & 95 & 9 & 101 & 36 & 131 \\
\hline
\end{tabular}

For the load-instructions, we keep track which data they have loaded. Basically, this means every word in memory has a pointer to the instruction which has loaded it. Upon a hit we decrease a per instruction miss-counter for the instruction that has previously loaded the requested data at a given address. Upon a read/write miss we increase the counter. Load-instructions with a misscounter value higher than zero, meaning that the instruction does not introduce any cache hit, are considered as special instructions.

For store-instructions the miss-counter is computed differently. We keep track which instructions have stored data to a particular address in memory. If upon loading a miss is detected, we increase the miss-counter for every previous storeinstruction, deploying a decaying penalty for older entries. Currently, we start with a penalty of 10 and halve it for every following older entry until we reach a value of 1 . Similar to the load instructions we use a threshold value of zero, meaning that higher values lead to a designation as a special store instruction.

Table 1 shows sample analysis results with four different applications on three different processor architectures. For each application, we counted the instructions in total, the number of load/stores, and the number of special load/store instructions chosen by the analysis algorithm. In general more special store instructions are picked than load instructions and the number of special instructions is not high in contrast to the total number of loads/stores. However, even with this small set of special instructions applications benefit from the Ihint approach. In the next section we show the experimental results.

\subsection{Cache Simulation}

In order to verify the proposed approach as well as to observe the influence of this novel design on performance we developed a cache simulator. Similar to any existing cache models, our tool simulates the basic functionality of the cache 
memory and provides statistics on cache hits and misses. The cache configuration can be specified by the user. An additional function of this cache simulator is to process the special load/store instructions. Hence, it requires not only the common memory access trace but also the instruction trace.

The right diagram in Fig. 2 illustrates the simulation process. As shown in the diagram, the simulator takes the memory access trace, the instruction trace, and the addresses of special instructions as input. For each memory access it models the lookup procedure and thereby modifies the status of the cache line. If the memory access is related to a special instruction, the simulator does not load the data into the cache for load operations or puts the data into the FIFO buffer for write operations. During the simulation procedure, the number of cache hits/misses and the amount of data transfers to the main memory are calculated.

For accuracy we adopted the dinero 4] cache simulator to verify our own cache simulation component. We compare some report of both simulators to be sure that our component provides correct information.

\section{Experimental Results}

The Ihint concept was verified using four applications of MediaBench 11] and MiBench 7]. These applications are PEGWIT, CJPEG, GSM, and DIJKSTRA. Each benchmark was run on MIPS, SPARC and PowerPC. PEGWIT deals with the cryptographic key generation; CJPEG handles the JPEG encoding; GSM works with audio decoding, and DIJKSTRA computes the shortest path between two nodes in a graph. We measured the amount of off-chip data traffic and the CPU cycles for data accesses. The first metric can be used to evaluate the energy consumption [15], while the second metric represents the performance.

All simulated caches are $4 \mathrm{x}$ associative and have 32 byte cache line size. Offchip memory accesses take 64 cycles for the first access and 32 cycles in burst mode for both reading and writing. Figure 3 depicts the experimental results, where we compare the metric values of Ihint with systems without and with caches.

We first observe all diagrams on the left side of the figure for performance issues. Overall, Ihint outperforms the normal cache in all benchmarks except for GSM, where both kind of caches behave similarly. This result holds true for all architectures; although they show different values due to the concrete design in instruction sets. Totally, CJPEG achieves an average performance improvement of $78 \%$ to the non-cache case and $43 \%$ to the normal cache on all three architectures. DIJKSTRAT introduces the best performance, where the Ihint cache works 3.3-folds better than the normal cache. With PEGWIT the performance gain is $52 \%$ and $72 \%$ individually. It is also surprised to see that with this application the cache version requires more time to access the data than the non-cache case. From the corresponding diagram on the right side it can be seen that a large amount of data are transferred from the cache to the off-chip memory. This means that for PEGWIT the additional traffic caused by the cache 


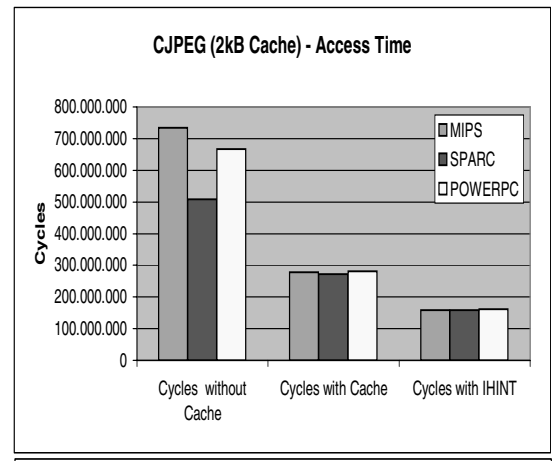

DIJKSTRA (2kB Cache) - Access Time
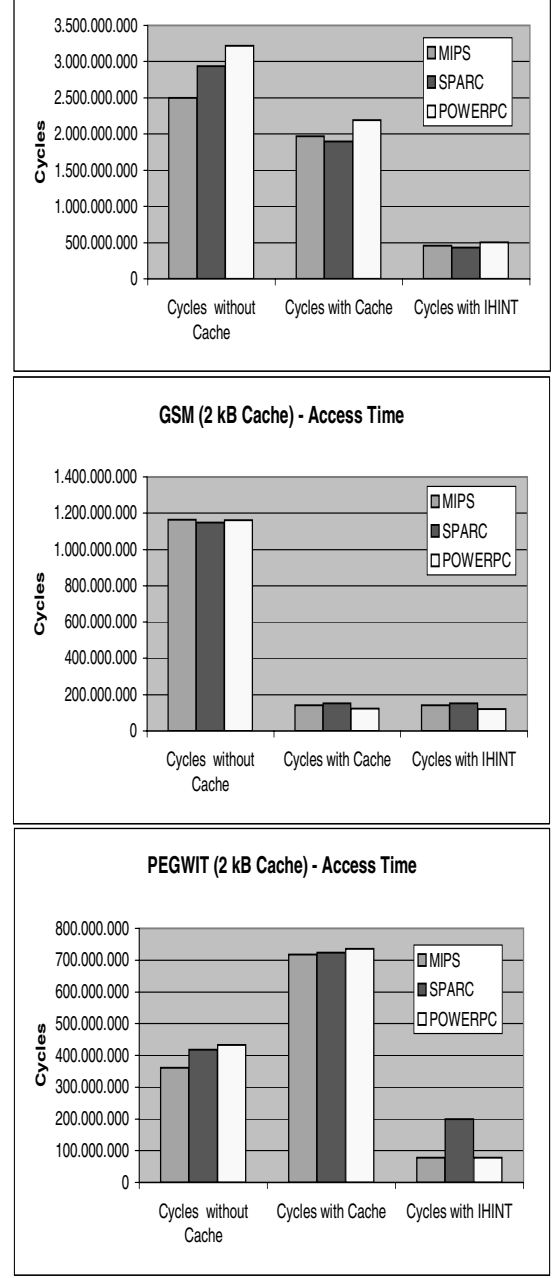

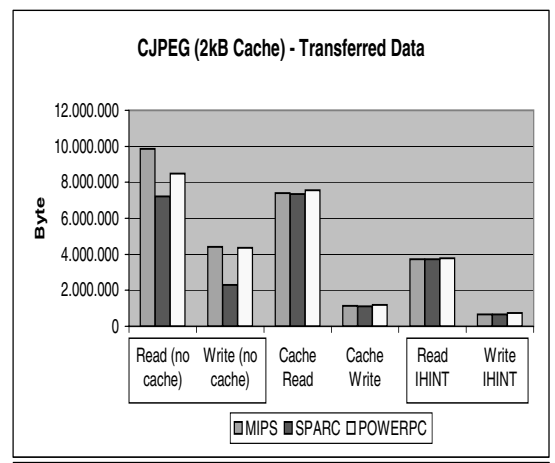

DIJKSTRA (2kB Cache) - Transferred Data

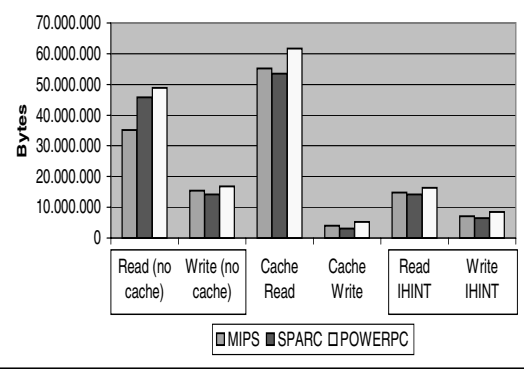

GSM (2 kB Cache) - Transferred Data

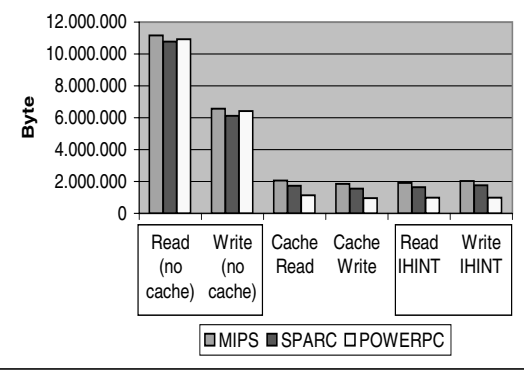

PEGWIT (2 kB Cache) - Transferred Data

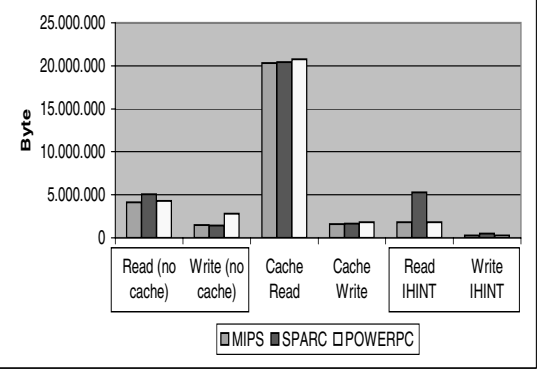

Fig. 3. Experimental results 
for implementing the cache policies exceeds the amount of data transfers in the non-cache case. As a result, the introduction of a cache memory does not bring benefit for this application. The Ihint cache, however, can better use the cache space, hence showing its advantages. With GSM Ihint fails to surpass the cache. This is because the normal cache already performs fairly well with a very low penalty for data access. In this case, the improvement space is limited.

Observing the diagrams on the right side of Fig. 3 similar results with the data traffic can be seen as with the access time: all applications, except GSM, show less data transfers with the Ihint approach. The decrease in data traffic indicates a decrease in power requirement. Unfortunately, we do not have an energy model to quantify the amount of power saving.

\section{Conclusions}

In this paper, we demonstrate an approach of selective bypassing of the cache memory based on instruction hints. It can be seen that our cache extension is able to exploit the new data paths for reading and writing to/from off-chip memory. Our analysis method is successful in selecting suitable load/store instructions at design time. As a result we achieve a much higher efficiency than a normal cache design.

The proposed approach was evaluated on embedded systems with small caches. In the next step, commodity caches with large size and realistic applications will be investigated. It is expected that the same gain can be achieved. In addition, we are also thinking about a hardware approach of selecting the special instructions.

\section{References}

1. Abella, J., González, A., Vera, X., O’Boyle, M.: IATAC: a smart predictor to turnoff L2 cache lines. ACM Transactions on Architecture and Code Optimization 2(1), 55-77 (2005)

2. ArchC. Archc - architecture description language (2007)

3. Benitez, D., Moure, J.C., Rexachs, D.I., Luque, E.: Evaluation of The Fieldprogrammable Cache: Performance and Energy Consumption. In: CF 2006: Proceedings of the 3rd conference on Computing frontiers, pp. 361-372 (2006)

4. Edler, J., Hill, M.D.: Dinero IV cache simulator

5. Esakkimuthu, G., Vijaykrishnan, N., Kandemir, M., Irwin, M.J.: Memory System Energy: Influence of Hardware-software Optimizations. In: ISLPED 2000: Proceedings of the 2000 international symposium on Low power electronics and design, pp. 244-246 (2000)

6. Gordon-Ross, A., Vahid, F., Dutt, N.: Fast Configurable-cache Tuning with a Unified Second-level Cache. In: ISLPED 2005: Proceedings of the 2005 international symposium on Low power electronics and design, pp. 323-326 (2005)

7. Guthaus, M.R., Ringenberg, J.S., Ernst, D., Austin, T.M., Mudge, T., Brown, R.B., Mibench: A free, commercially representative embedded benchmark suite. In: 2001 IEEE International Workshop on WWC-4. WWC 2001: Proceedings of the Workload Characterization, pp. 3-14 (2001) 
8. Hu, J., Kandemir, M., Vijaykrishnan, N., Irwin, M.J.: Analyzing data reuse for cache reconfiguration. Transactions on Embedded Computing System 4(4), 851876 (2005)

9. Ishihara, T., Fallah, F.: A Non-uniform Cache Architecture for Low Power System Design. In: ISLPED 2005: Proceedings of the 2005 international symposium on Low power electronics and design, pp. 363-368 (2005)

10. Kandemir, M., Kadayif, I., Choudhary, A., Zambreno, J.A.: Optimizing Inter-nest Data Locality. In: CASES 2002: Proceedings of the 2002 international conference on Compilers, architecture, and synthesis for embedded systems, pp. 127-135 (2002)

11. Lee, C., Potkonjak, M., Mangione-Smith, W.H.: Mediabench: a tool for evaluating and synthesizing multimedia and communicatons systems. In: MICRO 30: Proceedings of the 30th annual ACM/IEEE international symposium on Microarchitecture, pp. 330-335 (1997)

12. Pingali, V., McKee, S., Hseih, W., Carter, J.: Computation Regrouping: Restructuring Programs for Temporal Data Cache Locality. In: ICS 2002: Proceedings of the 16th international conference on Supercomputing, pp. 252-261 (2002)

13. Sermulins, J., Thies, W., Rabbah, R., Amarasinghe, S.: Cache Aware Optimization of Stream Programs. In: LCTES 2005: Proceedings of the 2005 ACM SIGPLAN/SIGBED conference on Languages, compilers, and tools for embedded systems, pp. 115-126 (2005)

14. Shen, X., Gao, Y., Ding, C., Archambault, R.: Lightweight Reference affinity analysis. In: ICS 2005: Proceedings of the 19th annual international conference on Supercomputing, pp. 131-140 (2005)

15. Sotiriadis, P.P., Chandrakasan, A.P.: A bus energy model for deep submicron technology. IEEE Trans. Very Large Scale Integr. Syst. 10(3), 341-350 (2002)

16. Yang, C., Lee, C.: HotSpot Cache: Joint Temporal and Spatial Locality Exploitation for I-cache Energy Reduction. In: ISLPED 2004: Proceedings of the 2004 international symposium on Low power electronics and design, pp. 114-119 (2004) 